\title{
Update: Selective adrenal venous sampling (AVS) - Indication, technique, and significance
}

\section{Update: Selektive Nebennierenvenenblutentnahme (NNVBE) - Indikation, Technik und Stellenwert}

Authors

Christina Loberg ${ }^{1}$, Gerald Antoch ${ }^{1}$, Johannes Stegbauer ${ }^{2}$, Till Dringenberg ${ }^{3}$, Andrea Steuwe ${ }^{1}$, Günter Fürst $^{1}$, Matthias Haase ${ }^{3}$, Lars Christian Rump², Lars Schimmöller ${ }^{1}$

Affiliations

1 University Dusseldorf, Medical Faculty, Department of Diagnostic and Interventional Radiology, Dusseldorf, Germany

2 Department of Nephrology, Medical Faculty, HeinrichHeine-University Düsseldorf, Düsseldorf, Germany

3 Division for Specific Endocrinology, University Hospital Düsseldorf, Düsseldorf, Germany

Key words adrenal gland, interventional procedures, angiography, blood, hypertension

received 03.07.2020

accepted 19.10.2020

published online 16.12.2020

Bibliography

Fortschr Röntgenstr 2021; 193: 658-666

DOI 10.1055/a-1299-1878

ISSN 1438-9029

(C) 2020. Thieme. All rights reserved.

Georg Thieme Verlag KG, Rüdigerstraße 14,

70469 Stuttgart, Germany

Correspondence

Priv.-Doz. Dr. med. Lars Schimmöller

University Dusseldorf, Medical Faculty, Department of

Diagnostic and Interventional Radiology, Moorenstraße 5,

40225 Düsseldorf, Germany

Tel.: $+49 / 211 / 8117754$

Fax: $+49 / 211 / 8116145$

lars.schimmoeller@med.uni-duesseldorf.de

\section{ABSTRACT}

Background Primary aldosteronism (PA) is the most common detectable cause of secondary hypertension. The majority of patients have either an adrenal aldosterone-producing adenoma (APA) or bilateral adrenal hyperplasia (BAH) demanding different therapeutic approaches. Screening tests and imaging cannot reliably distinguish between a unilateral or bilateral PA.
Methods This review article gives an overview concerning etiology, diagnostics, and therapeutic options of PA, and reviews the indication, the technique, and relevance of selective adrenal venous sampling (AVS) in the context of the current literature and the authors' experience.

Results AVS can verify or exclude a unilaterally dominated secretion with a high success rate. Patients with PA and a unilateral APA can be treated curatively by adrenalectomy.

Conclusions AVS is an established diagnostic examination for differentiation of unilateral from bilateral adrenal disease in patients with PA.

\section{Key Points:}

- Selective adrenal venous sampling (AVS) is a safe, reliable, and minimally invasive method to detect a unilateral or bilateral adrenal adrenal gland disease.

- Verification of lateralization by AVS has direct therapeutic relevance for patients with primary aldosteronism (PA).

- AVS can be performed with low radiation exposure, without contrast medium, and with a high success rate when performed by an experienced interventional radiologist.

\section{Citation Format}

- Loberg C, Antoch G, Stegbauer J et al. Update: Selective adrenal venous sampling (AVS) - Indication, technique, and significance. Fortschr Röntgenstr 2021; 193: 658-666

\section{ZUSAMMENFASSUNC}

Hintergrund Der primäre Hyperaldosteronismus (PHA; ConnSyndrom) ist der häufigste nachweisbare Grund für einen sekundären arteriellen Hypertonus. Primäre Ursachen hierfür sind das Aldosteron-produzierende Adenom (APA) oder eine bilaterale adrenale Hyperplasie (BAH) mit jeweils unterschiedlicher therapeutischer Konsequenz. Weder Screening- und Funktionstests noch die Schnittbildgebung können eine unilateral dominante Hormonproduktion nachweisen.

Methode Neben einer klinischen Übersicht über Ätiologie, Diagnostik und Therapie des PHA werden in diesem Übersichtsartikel die Indikation, die Technik und der Stellenwert der selektiven Nebennierenvenenblutentnahme (NNVBE) ausführlich dargestellt und im Kontext aktueller Literatur diskutiert. 
Ergebnisse Die NNVBE kann mit hoher Erfolgsrate eine unilaterale Dominanz nachweisen oder ausschließen. Patienten mit PHA und unilateralem APA können kurativ durch eine Adrenalektomie behandelt werden.
Schlussfolgerung Die NNVBE ist eine etablierte Intervention zur Subtypisierung des PHA in eine unilaterale oder bilaterale Erkrankung der Nebenniere.

\section{Introduction}

Primary hyperaldosteronism (PHA; Conn syndrome) results from autonomous aldosterone production in the adrenal cortex and, depending on the cohort of hypertensive patients studied, is the most common detectable cause of secondary arterial hypertension with a frequency of up to $15 \%[1,2]$. In addition, kidney disease, renal artery stenosis, Cushing syndrome or pheochromocytoma can cause secondary hypertension [3]. The most common causes of PHA are aldosterone-producing adenoma (APA) or bilateral adrenal hyperplasia (BAH) [4]. Aldosterone is produced in the zona glomerulosa of the adrenal gland and binds to the mineralocorticoid receptor in the distal tubule and collection tube of the kidney, which induces the increased incorporation of sodium channels $(\mathrm{ENaC})$ into the luminal membrane via transcription factors which regulate the reabsorption of salt and water. $\mathrm{PHA}$ is clinically manifested by arterial hypertension, possibly in combination with hypokalemia and metabolic alkalosis. More rarely, $\mathrm{PHA}$ is accompanied by hypernatremia and hypervolemia [5]. Patients with systolic blood pressure above $160 \mathrm{mmHg}$, refractory hypertension, hypertension with incidentaloma or family history of hypertension should be evaluated clinically for PHA [6]. The occurrence of PHA is associated with a significantly increased risk of cardiovascular disease [7-9].

Patients with PHA and confirmed unilateral disease with corresponding asymmetric aldosterone release can be treated curatively by surgical removal of the adrenal glands (adrenalectomy). If there is no lateral difference in aldosterone secretion, therapy is usually performed using an aldosterone antagonist (e. g. spironolactone, eplerenone) and possibly complementary anti-hypertensives. Bilateral adrenalectomy is not recommended [10, 11]. The high importance of AVS in the diagnostic sequence is illustrated below.

\section{Screening and Functional Test}

The detection of an elevated aldosterone-renin quotient (ARQ) (screening test) is indicative of PHA, but a number of drugs affect ARQ and should therefore be replaced with a non-interfering medication ahead of time [12]. The gold standard is a pathological saline load test. Intravenous administration of $2000 \mathrm{ml}$ of $0.9 \%$ saline solution over 4 hours suppresses serum aldosterone levels depending on physiological conditions (limits may vary according to test modality). Less frequently, a fludrocortisone suppression test (time-consuming) and captopril test (alternative for contraindications to volume administration) are used $[6,13]$. Physiologically expected aldosterone suppression is also absent after administration of fludrocortisone. Related tests have sensitivities and specificities between 80 and $90 \%$ [13]. An orthostasis test can be useful in differentiating between APA and $\mathrm{BAH}$, but is also rarely used in clinical practice. A constant or falling aldosterone value in the orthostasis test with evidence of an adrenal mass in the imaging would indicate APA with a certain probability, but this is not conclusive. In addition to the screening and functional tests, tomography is always recommended to assess the adrenal glands [14].

\section{Imaging}

Imaging of the adrenal glands is usually performed using computed tomography (CT) or magnetic resonance imaging (MRI). Such imaging allows the detection of uni- or bilateral macro- $(>1 \mathrm{~cm})$ or microadenomas $(\leq 1 \mathrm{~cm})$, bilateral macro- or micronodular hyperplasia or thickening of the adrenal gland or, in rare cases, adrenal carcinomas (less than $1 \%$ and usually $>4 \mathrm{~cm}$ ). Due to their high fat content, adenomas in native CT show average density values of $\leq 10$ Hounsfield units (HU) with more than 5-10\% pixels with negative density values [15]. After contrast medium (CM) administration, adenomas usually show a rapid absolute and relative wash-out, which means that even lipid-poor adenomas with density values up to $40 \mathrm{HU}$ can be detected with higher sensitivity [16]. In MRI, the characteristic fat detection can be done by chemical shift sequences, which distinguish fat- and hydrogen-bound protons within a voxel. If there is a relevant signal drop in the out phase (OP) compared to the in phase (IP), intracellular fat is present, demonstrating an adenoma. A Chemical Shift Index (CSI) [(signal intensity IP - signal intensity OP)/signal intensity IP $\times 100$ ] of over $20 \%$ has a high specificity for adrenal adenomas $[17,18]$.

However, neither CT nor MRI can differentiate between functional (hormone-active) and non-functional (hormone-inactive) adenomas. An increased number of hormone-inactive adrenal adenomas develop as a person ages, so that a unilateral adenoma or unilateral adrenal hyperplasia (UAH) is not automatically the cause of PHA. In contrast, microadenomas, which often escape imaging, can be hormone-active and cause secondary hypertension. Thus, unilateral aldosterone secretion may still be present in a supposedly morphologically normal adrenal gland or BAH. The detection or exclusion of a unilateral adrenal node using tomography is therefore usually not sufficient for a definitive therapeutic decision. A differentiation of the hormone-producing side is ultimately only possible through a side-separated AVS.

\section{Selective Adrenal Venous Sampling (AVS)}

\section{Indications}

AVS is an established procedure and represents the gold standard for the detection of unilateral or bilateral adrenal disease $[19,20]$. 
In addition, the diagnosis of ACTH-independent hypercortisolism (adrenal Cushing syndrome) is another indication for AVS [21].

\section{Adrenal Gland Anatomy}

The adrenal glands is located directly above the kidneys and are surrounded by the renal fat capsule. The venous outflow on the right side is directly into the inferior vena cava (IVC) usually dorsally on the right side via the right suprarenal vein (length 1 to $15 \mathrm{~mm}$ ). The left suprarenal vein (length 10 to $40 \mathrm{~mm}$ ) merges with the inferior phrenic vein mostly cranially ahead of the confluence with the left renal vein ( $\triangleright$ Fig. 1 ). In rare cases, adrenal veins may be 2- or 3-fold. Variants on the right are confluences in phrenic, intercostal, hepatic veins or directly into the right renal vein [22]. On the left-hand side, direct junctions into the IVC, into peripheral or segmental branches of the renal vein or a separate junction next to the phrenic vein have been described [23] $(\triangleright$ Fig. 2).

Imaging allows evaluation of the vascular anatomy, position of the adrenal glands and size of the IVC prior to a planned AVS. If adequate imaging was not performed, a native CT of the adrenal glands with a small slice thickness $(\leq 2 \mathrm{~mm})$ including sagittal and coronal image reconstructions should be supplemented. In most cases the inflow of the left adrenal vein into the renal vein is clearly visible. The right adrenal vein, on the other hand, can often not be detected exactly, therefore the height determination of the adrenal gland in relation to the vertebral bodies and the width of the IVC are important information for an AVS on this side.

\section{Technique and Performance of AVS}

As an interventional radiological procedure, AVS is subject to the usual requirements of patient education. As a venous intervention, AVS belongs to risk group 1 with a low risk of bleeding (Consensus Guidelines of CIRSE) [24]. The test should be performed in the morning due to the circadian fluctuations in aldosterone concentration, since aldosterone secretion correlates with renin secretion and shows the highest value in the morning hours. Since food intake can affect serum aldosterone concentrations, patients should remain fasting [25-27].

The literature describes procedures for AVS, which mostly differ in the selection of catheters (e. g. Cobra, Simmons, Vertebralis) and their sizes (4F, 5F, microcatheters), since there are no standard catheters available specifically for this intervention [20, 2830]. In addition to standardized catheter selection, the contrastfree procedure described below includes preparation of the catheter for the right side, which was already illustrated in 1996 [31]. This enabled us to achieve a hit rate of over $80 \%$ in a cohort of over 200 patients.

We perform the AVS using a sequential technique, i. e. sampling on both sides is performed successively via a one-sided access path ( $\triangleright$ Fig. 3 ). An antegrade puncture of the right common femoral vein medial to the arteries is routinely performed under sterile conditions under local anesthesia using a Valsalva maneuver with the introduction of a $6 \mathrm{~F}$ introducer sheath using the Seldinger technique. The $6 \mathrm{~F}$ sheath offers the advantage that peripheral blood can be drawn from the sheath despite the presence of the angiography catheter. For the left side, we use a
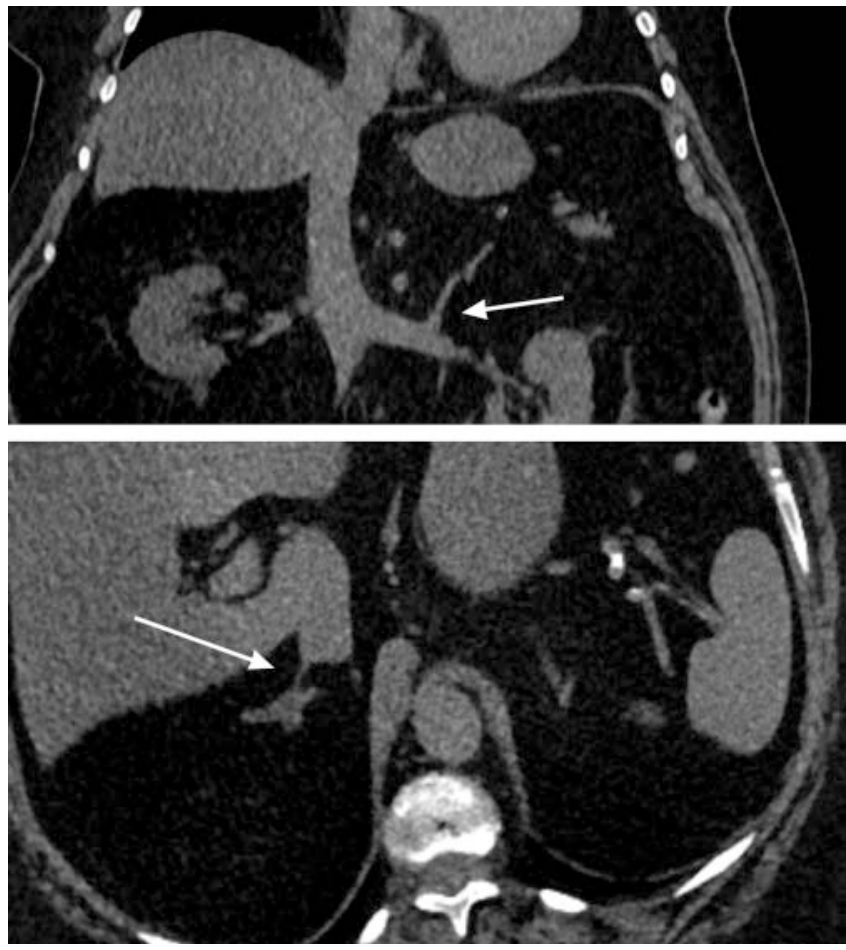

- Fig. 1 Native computed tomography (CT) with imaging of the adrenal veins in standard anatomy (top: inflow of the left adrenal vein (white arrow) in reconstructed curved coronal plane; bottom: right adrenal vein (white arrow) in reconstructed curved axial plane).

vertebral catheter due to its angled tip with a view to anatomy. A SIM-2 catheter or 4F Vertebralis catheter can be helpful as a second choice in the event of a steep angle of the left renal vein, severe scoliosis or renal vein atrophy. For the right side a Mickelson (MIK) catheter is advantageous due to its curvature and slightly raised tip. This allows the catheter tip to be inserted from above into the slightly caudal right adrenal vein. If the IVC is wide and there is no wall contact, a SIM-1 (IVC $>2.5 \mathrm{~cm}$ ) or SIM-2 catheter (IVC $>3 \mathrm{~cm}$ ) may be the second choice for the right side. With regard to blood collection, aspiration might be easier when using a $5 \mathrm{~F}$ catheter than with smaller catheters.

In our experience, it is recommended to manually provide the catheter for right-sided collection with an additional lateral hole to avoid collapse or occlusion of the fine adrenal vein during aspiration ( $\triangleright$ Fig.4) [31]. However, this procedure is an off-label use, as the perforation of an extra hole alters the medical device. Alternatively, an attempt can be made to obtain blood by draining from the catheter (without aspiration), but this is not always possible with a small adrenal vein.

Due to the better probing, we always start with collection from the left adrenal vein and normally use a $5 \mathrm{~F}$ Vertebralis catheter for this. The catheter is guided in the IVC via a slide-coated, configurable guide wire. The tip is rotated at the level of $L 1 / 2$ towards the left renal vein, so that the wire can be inserted as deeply as possible into the renal periphery; if the wire position is stable, it can then be used to insert the catheter. After the wire is removed, the catheter is rotated with the tip poised cranially and positioned with retraction in the ostium of the left adrenal vein (the "snap- 


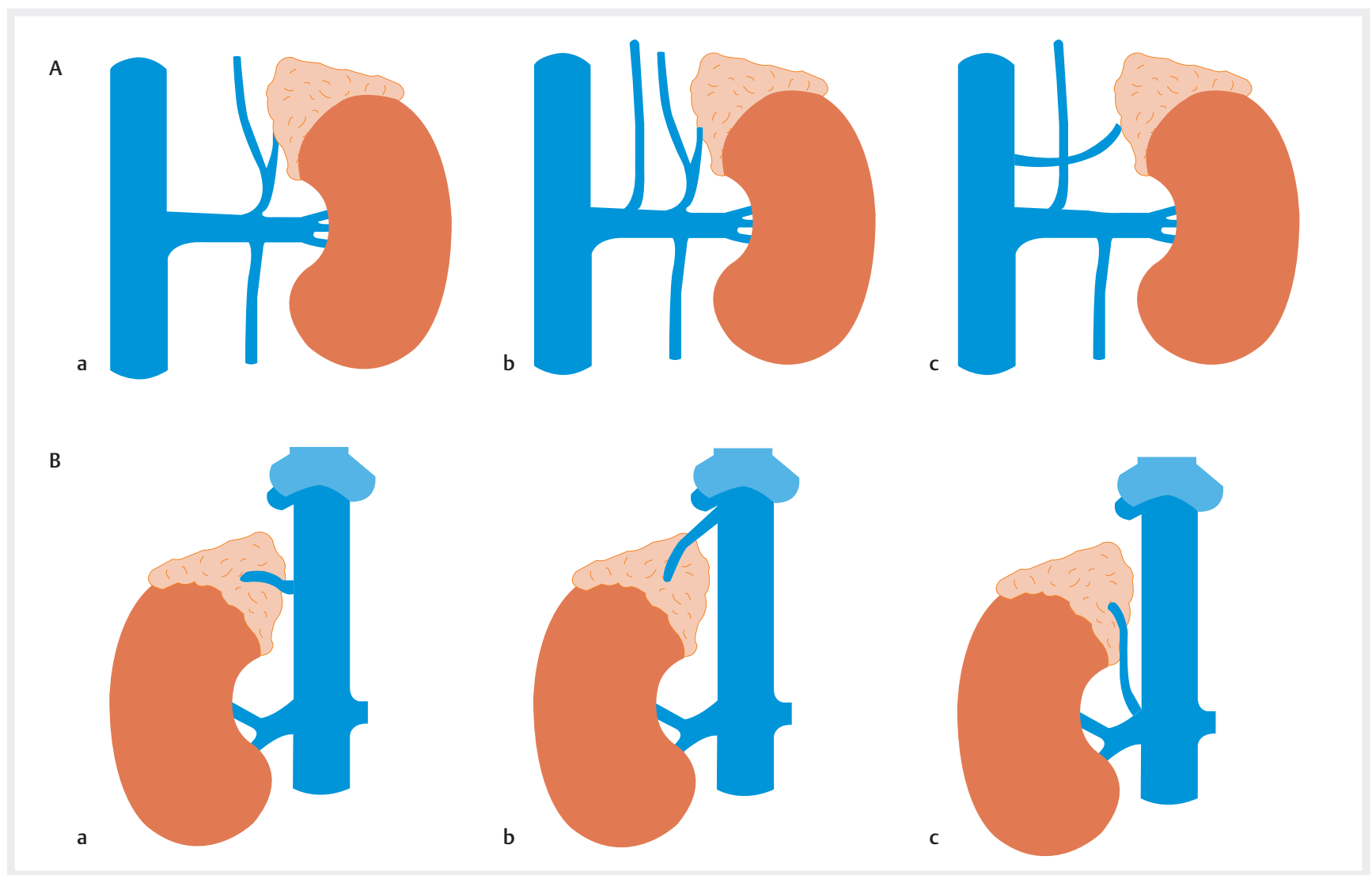

- Fig. 2 A Variants of left adrenal vein. a Standard anatomy. b Separate inflow of the inferior phrenic vein. c Direct inflow into the inferior vena cava. B Variants of the right adrenal veins. a Standard anatomy. $\mathbf{b}$ Inflow into the hepatic vein. $\mathbf{c}$ Inflow into the right renal vein.

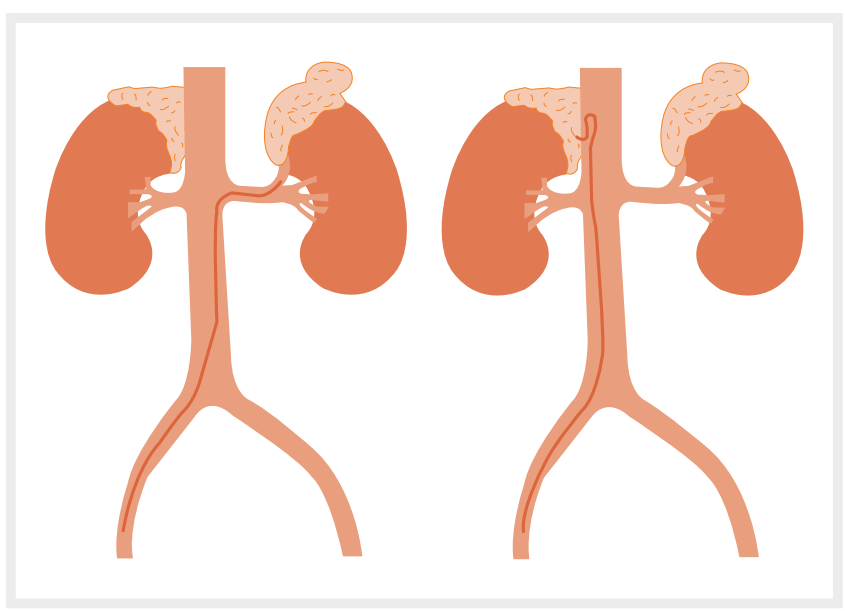

- Fig. 3 Graphic of catheter positions for unilateral access in sequential technique.

ping" is usually visible). In anterior-posterior fluoroscopy, the junction is usually at the level of the left-lateral edge of the vertebral body. Exploratory difficulties may arise in the case of scoliosis with an altered vascular angle or due to anatomical variants (e.g., outlet variants, renal agenesia or atrophy) ( Fig. 5-7).
The probing of the right adrenal vein, which usually opens directly into the IVC about $1 \mathrm{~cm}$ cranially of the renal vein, is usually more difficult. We use a 4 or 5F MIK catheter. The rear wall of the IVC should be searched slowly, observing breathing excursions from the height of the hepatic cap to the height of $L 1 / 2$. It is recommended to guide the catheter systematically from cranial to caudal at $15^{\circ}$ angles (dorsal left: $30^{\circ}$ and $15^{\circ}$; dorsal: $0^{\circ}$; dorsal right: $15^{\circ}, 30^{\circ}, 45^{\circ}, 60^{\circ}$ and $75^{\circ}$ ). Most frequently, the orifice is probed approx. $30^{\circ}$ right dorsally in the intercostal space at the level of T11/12. Aggravating factors on the right side can be especially a wide IVC, significant breathing excursions or the small size of the adrenal vein.

In difficult cases a dose of contrast can support probing ( $\triangleright$ Fig. 8). Some authors advocate general contrast administration for identification of the adrenal vein, probing and/or position control. However, this involves the risk of stimulation of the adrenal gland and increases the likelihood of adrenal vein hemorrhage/damage by increasing pressure in the adrenal vein and consecutively in the adrenal gland during injection. If it is necessary to identify the adrenal vein by the administration of contrast medium, it is advisable to draw blood in advance and only then to administer contrast medium, which, if possible, should not be injected into the adrenal gland. In many cases, however, a reliable distinction is not possible even with contrast. In our view, a standard contrast medium dose is not necessary for a successful AVS and should be avoided whenever possible. Natively, $5 \mathrm{ml}$ of selec- 


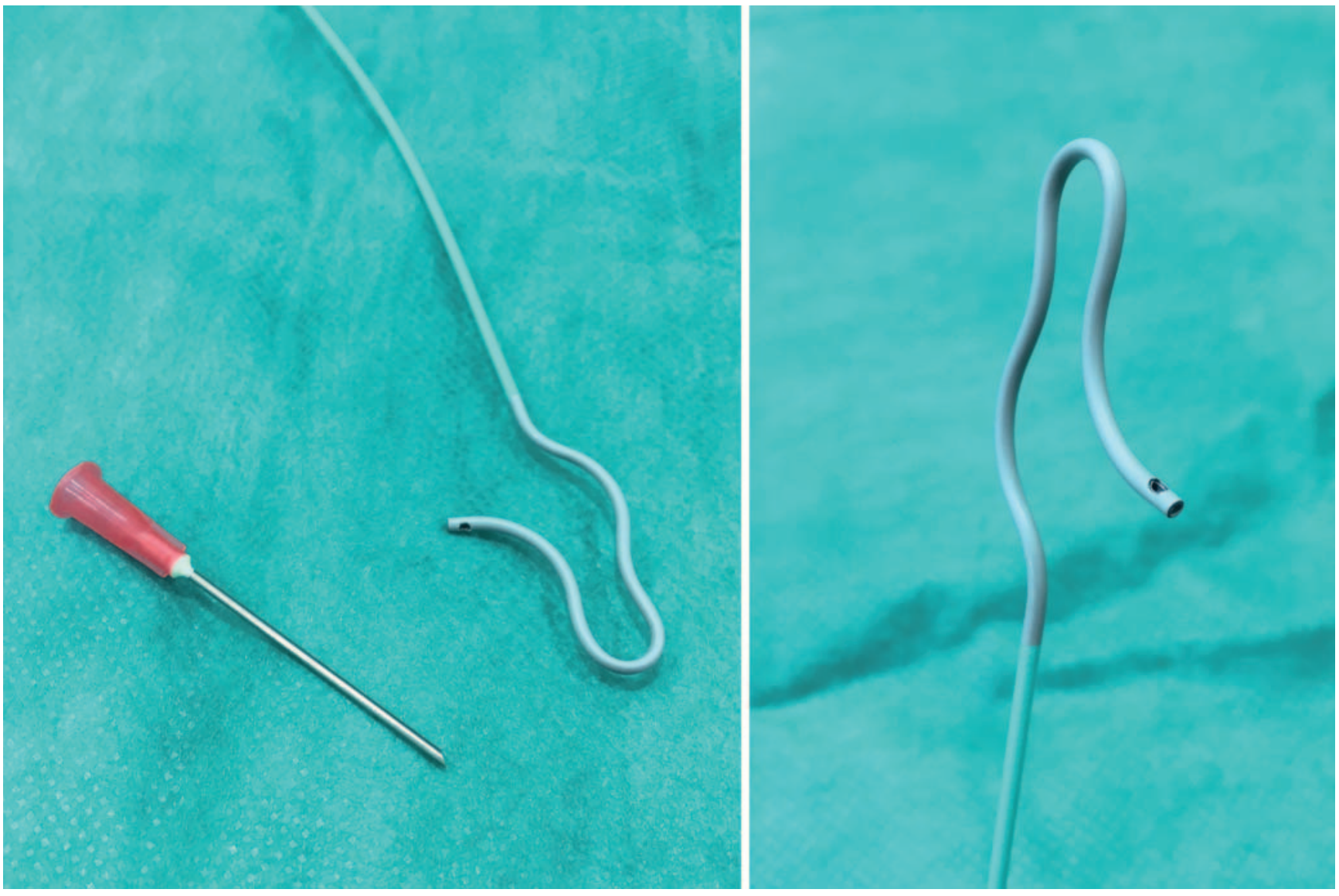

- Fig. 45 French Mickelson catheter (5F MIK) with prepared hole.

tive blood should be taken from all probeable outlets at different heights in the corresponding probing window, since e.g. accessory small hepatic veins also open. We take up to 6 samples from the right side and 3 samples from the left. Due to the time required (at least 25 minutes), a peri-interventional rapid cortisol test with determination of the cortisol in the plasma from the adrenal vein and the sheath could not be performed [20, 32].

In order to calculate the selectivity index, one or more peripheral samples must be taken in consultation with the referring physician (endocrinologist, nephrologist); there is no standardized, generally applicable acceptance protocol [33]. This can be done for example, from the common iliac vein via the sheath or from the IVC. After a final check for completeness and correct labeling of the samples, the sheath is removed.

\section{Contraindications}

There are few contraindications for AVS. The procedure should not be performed if primary genetic causes such as familial hyperaldosteronism (Type I-IV) or PASNA (Primary Aldosteronism, Seizures and Neurologic Abnormalities) syndrome are not ruled out. In the case of diseases of the blood coagulation system, shortened life expectancy or other diseases that significantly restrict general health, an appropriate indication review should be performed. The INR value should be $\leq 2.0$ and the platelet count $\geq 50000$ per $\mu$ l. Taking anticoagulants is generally not a contrain- dication in Risk Group 1. Performance of AVS should certainly be discussed with patients who general refuse surgical removal of an adrenal gland. Contrast medium allergy is not a contraindication in native examination techniques.

\section{Complications}

When performed by experienced physicians, AVS represents a very low complication rate [34]. In individual cases, the inguinal puncture may be more difficult, e. $\mathrm{g}$. if there is an anatomical positional variance (however, an AV fistula is extremely rare). The catheter can potentially injure the veins, especially the small adrenal veins; likewise, hemorrhaging into the adrenal glands is possible [35]. Clinically-related thrombosis or emboli are unlikely. In addition to an allergic reaction when contrast is applied, another possible complication is bleeding into the adrenal gland if the injection pressure is too high.

\section{Post-AVS Laboratory Analysis}

The selectivity index (SI: adrenal vein cortisol $[\mathrm{AV}] /$ peripheral vein cortisol [PV]) is used to determine the hit rate in the selective blood samples. A quotient $\geq 2$ evaluates the corresponding selective removal as positive (specificity $100 \%$ ) [26]. A quotient $<2$ therefore does not formally correspond to a selective AVS. It should be noted that the level of the selectivity quotient thus directly affects the sensitivity and specificity of the AVSE [26]. 

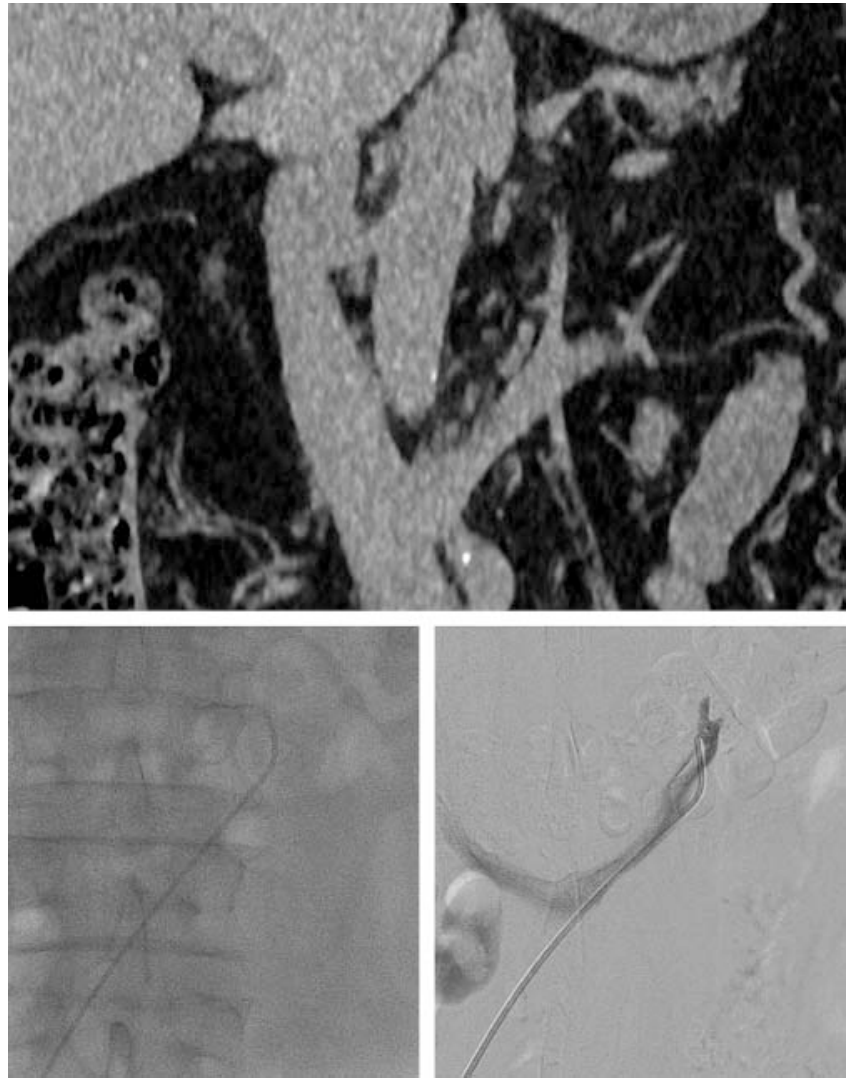

- Fig. 5 Left AVS: illustration of atypical outlet of left renal vein at $\mathrm{L} 4 / 5$ ( $5 \mathrm{~F}$ Vertebralis catheter).
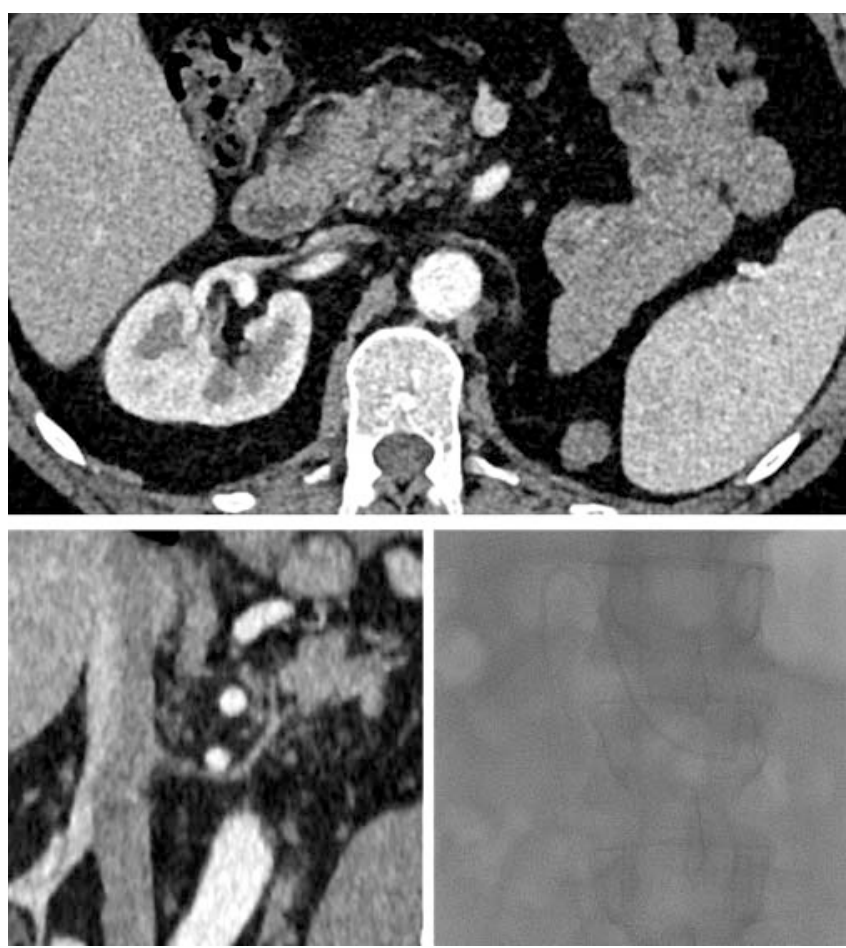

- Fig. 6 Left AVS: Left renal agenesia with absent left kidney and narrow left renal vein (4F SIM-2 catheter).
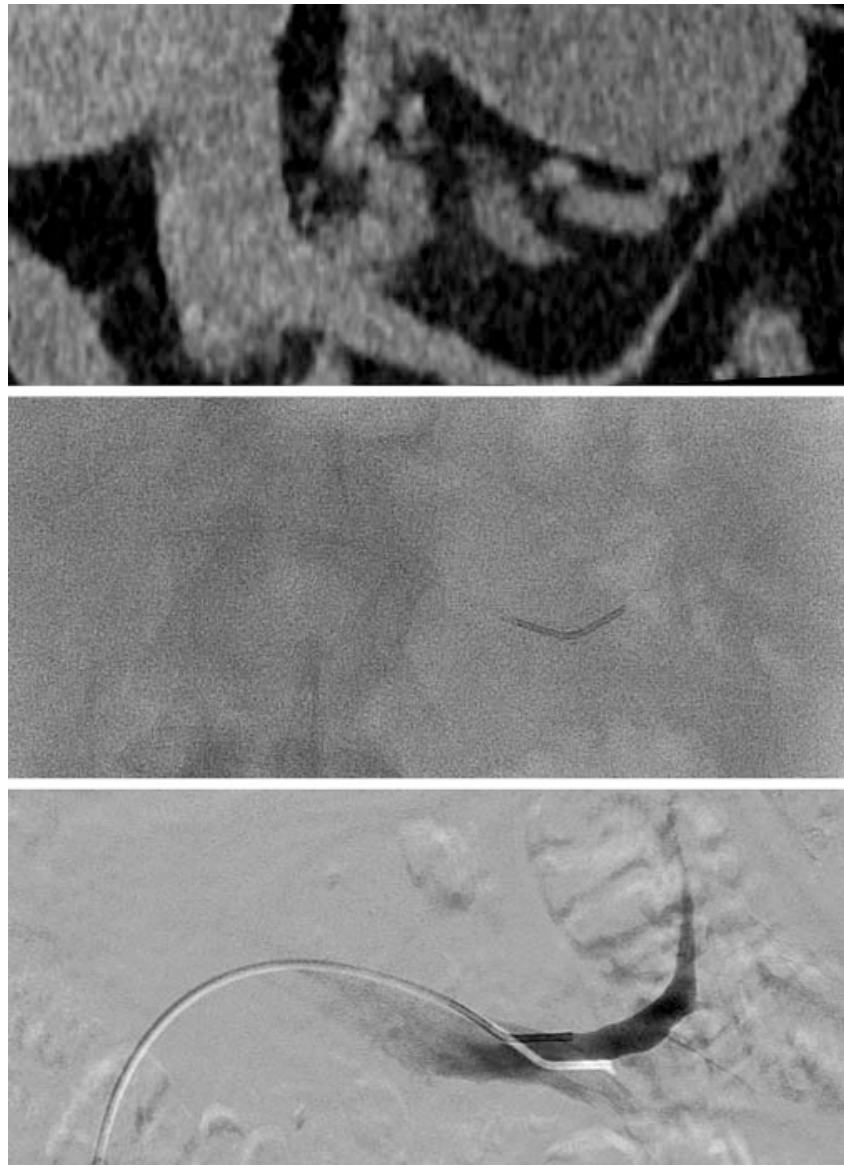

- Fig. 7 Left AVS: Atypical outlet of left adrenal vein peripherally from the renal vein ( $5 \mathrm{~F}$ Vertebralis catheter).

Contamination with peripheral blood or an overall reduced concentration can distort the results. To prove lateralization, the aldosterone cortisol quotient (ACQ) must be compared for both adrenal veins (aldosterone AV side 1 /cortisol AV side 1 versus aldosterone AV side 2/cortisol AV side 2). If there is a quotient $\geq 3$ in favor of one side, there is a high probability that the corresponding side has an APA. If selective AVS is successful, the sensitivity and specificity are over $95 \%$ [36]. It should be noted, however, that sometimes differing limit values are used. The interpretation and the resulting therapy recommendation should not be dogmatic, but always take into account the overall clinical and diagnostic situation.

\section{Discussion}

AVS is a safe and very efficient method for sub-typing PHA as a unilateral or bilateral disease of the adrenal gland. In the case of unilateral dominance, an APA can be assumed even without evidence in cross-sectional imaging; in the absence of unilateral dominance, a BAH can be assumed. However, the result of the examination depends to a large extent on the experience of the interventionalist [37]. Probing and sampling from the right adrenal vein is technically more difficult than from the left and is the most common cause of an unsuccessful AVS. The technical suc- 


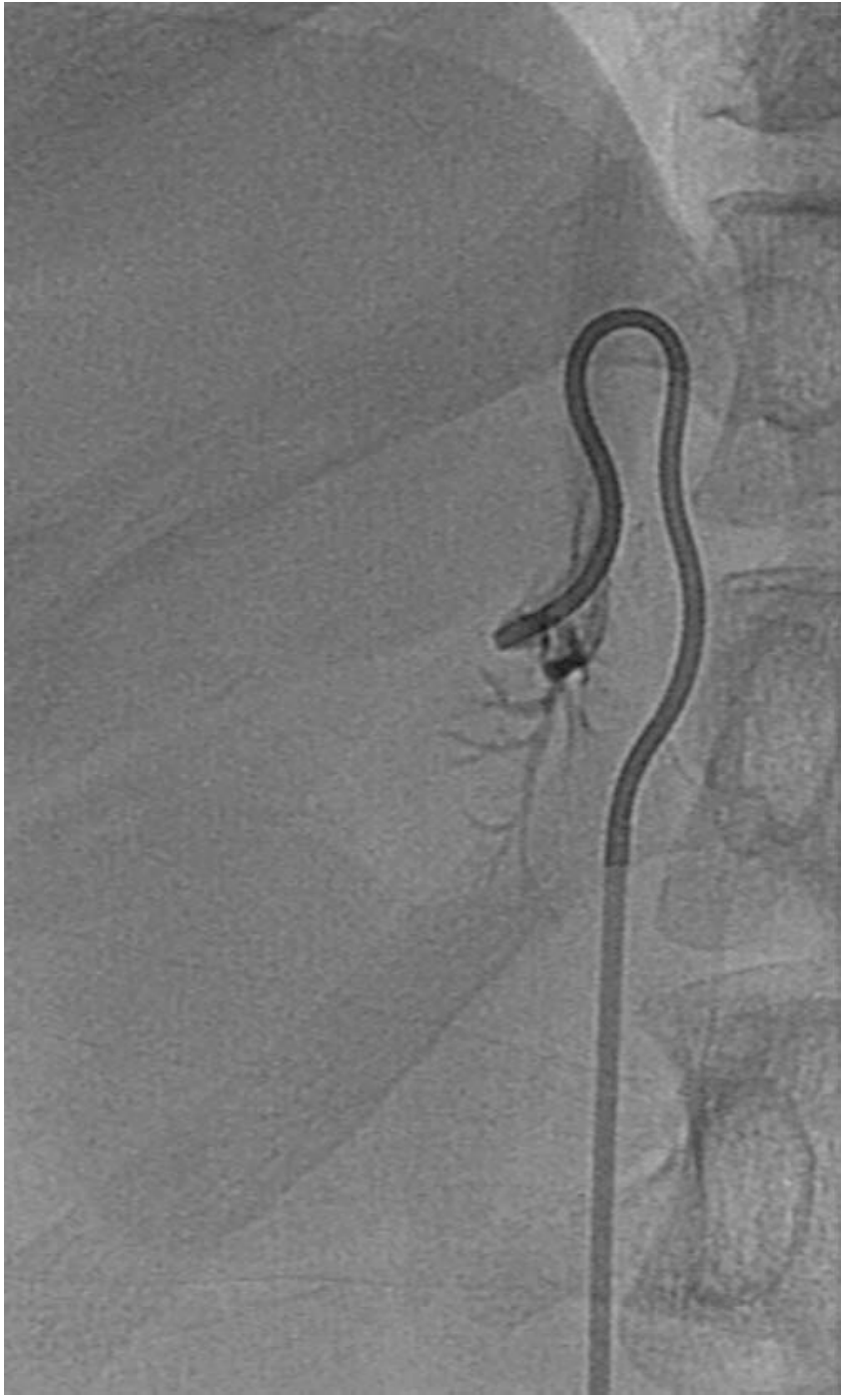

Fig. 8 Right AVS: Illustration of the right-side adrenal veins using DSA and contrast using a 5F MIK catheter. Standard anatomy, corresponding to direct confluence of the right adrenal vein into the IVC.

cess rate in specialized centers, however, is stated to be up to $90 \%$ $[28,38]$. In contrast, with an optimal selectivity index of 2 , sensitivities of a maximum of $71 \%$ are given [25]. In our institute we achieve a bilateral success rate of over $80 \%$ using the native procedures described above (left $>90 \%$; right $>80 \%$ ). A bilateral hit rate of $70-90 \%$ should be targeted. The result of a unilaterally successful AVS cannot be used diagnostically with certainty, so that the procedure should be repeated at least once (over $50 \%$ chance of success). A thin-slice CT with IV contrast can be helpful to clarify anatomical norm variants.

Simultaneous AVS with bilateral puncture and insertion of two angiography catheters with simultaneous bilateral blood sampling to achieve better results are significantly more time-consuming for both the patient and interventionalist and have not been established over the sequential technique in routine practice [39].
In addition to standard pure lateralization diagnostics, individual hyperfunctional areas of the adrenal gland can also be selectively differentiated from different adrenal sub-segments by segmental blood collection using specialized microcatheters [29, 40]. Clinically, however, differentiation at the segmental level is usually not relevant, with a poor cost-benefit ratio.

Various centers perform pre-interventional stimulation with administration of a synthetic peptide of the adrenocorticotropic hormone (ACTH) (Synacthen $50 \mu \mathrm{g} / \mathrm{h})$ [41]. In the case of stimulation, however, it should be noted that the limit values for the selectivity and lateralization indices shift. There is no clear evidence that stimulation with ACTH improves diagnosis and therefore it is not standardly performed $[42,43]$.

Depending on experience and the technique used, there are significant differences in radiation exposure among centers [29, $38,39]$. In our clinic the median fluoroscopy time and radiation exposure is 16 minutes and less than $4 \mathrm{mSv}$ due to the native, standardized implementation in the lower range of the literature values (fluoroscopy times 16-27 min; effective dose 3.2-29 mSv) [38]. Some authors recommend performing a cone beam CT for easier probing of the adrenal veins, which, however, increases radiation exposure $[44,45]$. Significantly lower dose exposure can be achieved by means of pulsed fluoroscopy, which is particularly important in view of the sometimes younger patients.

Based on our own experience in only less than $50 \%$ correlated cross-sectional imaging, a relevant proportion of patients (about $30 \%$ ) can be given a curative adrenalectomy by AVS even without adenoma detection in imaging. Detectable adenomas can thus be functionally lateralized in around $40 \%$ of cases. A diagnosis based solely on cross-sectional imaging alone is therefore not sufficient in most cases [46]. The SPARTACUS study published in The Lancet in 2016, which showed that a treatment decision based on CT was not inferior to AVS within a 1-year follow-up, is discussed controversially, particularly with regard to study-related limitations $[47,48]$. AVS continues to be the gold standard in the guidelines [49]. However, the results of selective AVS should always be assessed in the context of sectional imaging diagnosis and clinical symptoms $[5,50]$.

Surgical therapy in case of confirmed lateralization is still the therapy of choice for PHA [51]. Interventional radiological therapy options, such as radio frequency ablation, may in the future represent an alternative therapeutic approach for multi-morbid or elderly patients, [52].

\section{Conclusion}

AVS is a low-risk procedure with high sensitivity and specificity for the identification of lateralized disease and is currently the gold standard in the clarification of PHA. Careful patient selection, pre-interventional planning using $\mathrm{CT}$ or MRI and an experienced interventionalist are required to achieve a high hit and success rate. The diagnostic added value of the method for the subsequent therapy decision is significantly increased compared to a clinical and imaging-based diagnosis alone. 


\section{ABREVIATIONS \\ ACQ aldosterone-cortisol ratio \\ ACTH adrenocorticotropic hormone \\ APA aldosterone-producing adenoma \\ ARR aldosterone-renin ratio (screening test) \\ AVS adrenal venous sampling \\ $\mathrm{BAH}$ bilateral adrenal hyperplasia \\ CM contrast medium \\ CSI chemical shift index \\ CT computed tomography \\ HU Hounsfield units \\ INR international normalized ratio \\ IVC inferior vena cava \\ MRI magnetic resonance imaging \\ $\mathrm{SI} \quad$ selectivity index \\ UAH unilateral adrenal hyperplasia}

\section{Conflict of Interest}

The authors declare that they have no conflict of interest.

\section{References}

[1] Rossi GP, Bernini G, Caliumi C et al. A prospective study of the prevalence of primary aldosteronism in 1125 hypertensive patients. J Am Coll Cardiol 2006; 48: 2293-2300

[2] Douma S, Petidis K, Doumas M et al. Prevalence of primary hyperaldosteronism in resistant hypertension: a retrospective observational study. Lancet 2008; 371: 1921-1926

[3] Young WF. Primary aldosteronism: renaissance of a syndrome. Clin Endocrinol 2007; 66: 607-618

[4] Heinrich DA, Adolf C, Rump LC et al. Primary Hyperaldosteronism: key characteristics at diagnosis: a trend toward milder forms. Eur J Endocrinol 2018; 178: 605-611

[5] Martell-Claros N, Abad-Cardiel M, Alvarez-Alvarenz B et al. Primary aldosteronism and its various clinical scenarios. J Hypertens 2015; 33: $1226-1232$

[6] Funder JW, Carey RM, Mantero F et al. The Management of Primary Aldosteronism: Case Detection, Diagnosis, and Treatment: An Endocrine Society Clinical Practice Guideline. J Clin Endocrinol Metab 2016; 101: 1889-1916

[7] Prejbisz A, Warchol-Celinska E, Lenders JW et al. Cardiovasc. Risk in Primary Hyperaldosteronism. Horm Metab Res 2015; 47: 973-980

[8] Rossi GP, Seccia TM, Maiolino G et al. The cardiovasculars consequences of hyperaldosteronism. Ann Endocrinol 2020 [online first]

[9] Pillai PR, Griffith M, Schwarcz MD et al. Primary Hyperaldosteronism: Cardiovasc. Risk, Diagnosis and Management. Cardiol Rev 2020; 28: 84-91

[10] Terzolo M, Stigliano A, Chiodini I et al. AME position statement on adrenal incidentaloma. Eur J Endocrinol 2011; 164: 851-870

[11] Chiloiro S, Giampietro A, Raffaelli M et al. Synchronous bilateral adrenalectomy in ACTH-dependent hypercortisolism: predictors, biomarkers and outcomes. Endocrine 2019; 66: 642-649

[12] Vonend O, Quack I, Rump LR. Aldosteron und Hypertonie. Wien klin Wochenschr 2010; 122: 65-74

[13] Song Y, Yang S, He W et al. Confirmatory Tests for the Diagnosis of Primary Hyperaldosteronism: A Prospective Diagnostic Accuracy Study.
Chonquing Primary Aldosteronism Study (CONPASS) Group. Hypertension 2018; 71: 118-124

[14] Delivanis DA, Vassiliadi DA, Tsagarakis S. Adrenal Imaging in Patients with Endocrine Hypertension. Endocrinol Metab Clin N Am 2019; 48: 667-680

[15] Boland GW, Lee M], Gazelle GS et al. Characterization of adrenal masses using unenhanced CT: an analysis of the CT literature. Am J Roentgenol 1998; 171: 201-204

[16] Korobkin M, Brodeur F], Francis IR et al. CT Time - attenuation washout curves of adrenal adenoma s and nonadenomas. Am J Roentgenol 1998; 170: 747-752

[17] Seo JM, Park BK, Park SY et al. Characterization of lipid - poor adrenal adenoma: chemical shift MRI and wash-out CT. Am J Roentgenol 2014; 202: 1043-1050

[18] Siegelman ES. Adrenal MRI: Techniques and clinical applications. L Magn Reson Imaging 2012; 36: 272-285

[19] Stowasser M, Gordon RD, Rutherford JC et al. Diagnosis and management of primary aldosteronism. J Renin Angiotensin Aldosterone Syst 2001; 2: 156-169

[20] Blondin D, Quark I, Haase M et al. Indikationen und Technik der selektiven Nebennierenvenenblutentnahme. RöFo 2015; 187: 19-28

[21] England RW, Geer EB, Deipolyi AR. Role of Venous Sampling in the Diagnosis of Endocrine Disorders. J Clin Med 2018; 7: 114

[22] Yeh MW, Duh QY. Chapter 41: The Adrenal Glands. In: Townsend CM Sabiston Textbook Of Surgery. Sauders: Elsevier; 2008. Available on https://thoracickey.com/the-adrenal-glands/

[23] Cesmebasi A, Du Plessis M, lannatuono $M$ et al. A review of the anatomy and clinical significance of adrenal veins. Clin Anat 2014; 27: 1253-1263

[24] Patel IJ, Davidson JC, Nikolic B et al. Consensus Guidelines for Periprocedural Management of Coagulation Status and Hemostasis Risk in percutaneous Image-guides Interventions. JVIR 2019; 30: 1168-1184

[25] Mailhot JP, Traistaru M, Soulez G et al. Adrenal Vein Sampling in Primary Hyperaldosteronism: Sensitivity and Specificity of Basal Cortisol and Aldosterone Ratios to Confirm Cathererization of the Adrenal Vein. Radiology 2015; 277: 887-894

[26] Ueland GA, Methlie P, Jøssang DE et al. Adrenal Venous Sampling for Assessment of Autonomous Cortisol Secretion. ] Clin Endocrinol Metab 2018; 103: 4553-4560

[27] Rossi GP, Auchus RJ, Brown M et al. An expert consensus statement on use of adrenal vein sampling for the subtyping of primary aldosteronism. Hypertension 2014; 63: 151-160

[28] Kahn SL, Angle F]. Adrenal Vein Sampling. Tech Vasc Interv Radiol 2010 13: $110-125$

[29] Satani N, Ota H, Seiji K et al. Intra-adrenal aldosterone secretion: Segmental adrenal venous sampling for localization. Radiology 2016; 278: 265-274

[30] Daunt N. Adrenal Vein Sampling: How to Make It Quick, Easy, and Successful. RadioGraphics 2005; 25: 143-158

[31] Doppmann JL, Gill JR. Hyperaldosteronism: Sampling the Adrenal Veins. Radiology 1996; 198: 309-312

[32] Cesari M, Ceolotto G, Rossitto G et al. The Intra-Procedural Cortisol Assay During Adrenal Vein Sampling: Rationale and Design of a Randomized Study (I-Padua). High Blood Press Cardiovasc Pre 2017; 24: $167-170$

[33] Funder JW, Carey RM, Mantero F et al. The Management of Primary Aldosteronism:Case Detection, Diagnosis, and Treatment:An Endocrine Society Clinical Practice Guideline. J Clin Endocrinol Metab 2016; 101: 1889-1916

[34] Rossi GP, Barisa M, Allolio B et al. The adrenal vein sampling international study AVIS for identifying the major subtypes of primary aldosteronism. I Clin Endocrinol Metab 2012; 97: 1606-1614 
[35] Monticone S, Satoh F, Dietz AS et al. Clinical Management and Outcomes of adrenal Hemorrhage Following Adrenal Vein Sampling in Primary hyperaldosteronism. Hypertension 2016; 67: 146-152

[36] Lim V, Guo Q, Grant CS et al. Accuracy of adrenal imaging and adrenal venous sampling in predicting surgical cure of primary aldosteronism. J Clin Endocrinol Metab 2014; 99: 2712-2719

[37] Vonend O, Ockenfels N, Gao X et al. Adrenal venous sampling: evaluation of the German Conn's registry. Hypertension 2011; 57: 990-995

[38] Fuss CT, Treitl M, Rayes $\mathrm{N}$ et al. Radiation exposure of adrenal vein sampling: a German Multicenter Study. Eur J Endocrinol 2018; 179: 261-267

[39] Lupi A, Battistel M, Barbiero G et al. Simultaneous bilateral adrenal vein sampling for primary aldosteronism: useful tips to make it simple and safe. Eur Radiol 2019; 11: 6330-6335

[40] Noda Y, Goshima S, Nagata S et al. Utility of microcatheter in adrenal venous sampling for primary aldosteronism. Br J Radiol 2020; 93: 1109

[41] Rossitto G, Maiolino G, Lenzini L et al. Subtyping of primary aldosteronism with adrenal vein sampling: Hormone- and side-specific effects of cosyntropin and metoclopramide. Surgery 2018; 163: 789-795

[42] Kline GA, So B, Dias VC et al. Catheterization during adrenal vein sampling for primary aldosteronism: failure to use (1-24) ACTH may increase apparent failure rate. J Clin Hypertens (Greenwich) 2013; 15 : 480-484

[43] Deinum J, Groenewoud H, van der Wilt G] et al. Adrenal venous sampling: cosyntropin stimulation or not? Eur J Endocrin 2019; 181: D15D26

[44] Onozawa S, Murata S, Tajima $\mathrm{H}$ et al. Evaluation of right adrenal vein cannulation by computed tomography angiography in 140 consecutive patients undergoing adrenal venous sampling. Eur J Endocrinol 2014; 170: 601-608

[45] Meyrignac O, Arcis É, Delchier MC et al. Impact of cone beam CT on adrenal vein sampling in primary aldosteronism. Eur ] Radiol 2020; 124 [online first]

[46] Ladurner R, Sommerey S, Buechner S et al. Accuracy of adrenal imaging and adrenal venous sampling in diagnosing unilateral primary aldosteronism. Eur J Clin Invest 2017; 47: 372-377

[47] Dekkers T, Prejbisz A, Kool LJ et al. Adrenal vein sampling versus CT scan to determine treatment in primary aldosteronism: An outcome-based randomised diagnostic trial. Lancet Diabetes Endocrinol 2016; 4: 739746

[48] Beuschlein F, Mulatero P, Asbach E et al. The SPARTACUS Trial: Controversies and Unresolved Issues. Horm Metab Res 2017; 49: 936-942

[49] Sk2-Leitlinie Operative Therapie von Nebennierentumoren. Langversion; Dezember 2017; AWMF-Register-Nummer: 088/008. https://www. awmf.org/leitlinien/detail/ll/088-008.html (Zugriff am: 22.08.2020)

[50] Tomaschitz A, Dobnig H. Primärer Hyperaldosteronismus - die häufigste monokausale Ursache der essenziellen Hypertonie. J Clin Endocrinology and Metabolism 2009; 2: 14-21

[51] Vorselaars WMCM, Nell S, Postma EL et al. Clinical Outcomes After Unilateral Adrenalectomy for Primary Aldosteronism. JAMA Surg 2019; 154: e185842

[52] Liang KW, Jahangiri Y, Tsao TF et al. Effectiveness of Thermal Ablation for Aldosterone-Producing Adrenal Adenoma: A Systematic Review and Meta-Analysis of Clinical and Biochemical Parameters. J Vasc Interv Radiol 2019; 30: 1335-1342 